\title{
Effects of concentration and synergism on drug solubilising behaviour of PVP K 30 and PEG 6000
}

\author{
Jannatul Fardous, Faria Farzana Perveen, Md. Ohidullah, A H M Saifuddin and \\ Sakina Sultana* \\ Department of Pharmacy, Jahangirnagar University, Savar, Dhaka-1342, Bangladesh
}

\begin{abstract}
The effect of concentration and synergism on drug solubilising behaviour of PVP K 30 and PEG 6000 were investigated. Our results showed that amount of drug solubilised by the surfactants decreased as surfactant concentration was increased. Regarding the effect of synergism of the two surfactants on drug solubilisation our results failed to show synergism. The amount of drug solubilised by a single surfactant did not differ much when combination of surfactants were used.
\end{abstract}

Key words: PVP K 30, PEG 6000, PEG 20000, Tween 80 and Theophylline.

\section{INTRODUCTION}

In modern medical sciences it is well reported that surfactant molecules play a pivotal role in enhancing apparent aqueous solubility of sparingly soluble and insoluble solutes through solubilisation technique. In recent times, therefore uses of surfactants in targeted drug therapy has been increased by many folds. The reason which is responsible for showing such performance is that surfactants are amphiphilic molecules with structural uniqueness. These molecules consists of a polar head moiety and a non polar tail part. These molecules because of such structural duality can form aggregates with definite shape and size in solution. Interestingly these molecules do not form aggregates all the times. Rather they form aggregates only when prerequisite conditions are met. Aggregates when become circular in shape are called 'micelles' and the prerequisite concentration is known as 'critical micellar concentration' (cmc) (Lieberman et al., 1988; Rawlins, 2004).

It is documented that micelles offer certain specificities. For instances these are consisted of three regions and these are micellar core, palisade layer and the outer surface. Micellar core is the inner portion, palisade layer is the junction area between the inner portion and outer regions and outer surface as name suggests is the outermost layer of the aggregates (Fig 1). Another specificity is that micelles offers varying polarity across their structure. If micelles are formed in aqueous media the outer surface would be polar and the inner core would be non polar; while in oil media the surface would be non polar and core would be polar. Now if a solute is present in the media then micelles offers sites of residence for that solute. Depending on the polarity of the solute, the solute can reside

\footnotetext{
*Corresponding author e-mail : sultana_s2003@yahoo.co.uk
} 
either on the surface or can be entrapped into the core. In general a hydrophobic solute gets physically (not molecular) entrapped inside the non polar core. Under such circumstances micelles acts like a micro-container. It carries the solute to the target sites escaping the hostile polar media where it is not miscible. This is solubilisation. Due to solubilisation the solubility of hydrophobic solute apparently increases in the polar media (Lieberman et al., 1988). A hydrophobic drug molecule also experiences the same in presence of a surfactant molecule.

It is reported that solubilisation process depends on several factors among which surfactant type, polarity of the incoming solute molecule, working concentration and local temperature are the major ones (Rawlins, 2004). In recent years although surfactant propelled drug solubilisation technique has been investigated a lot but interestingly regarding the effects of surfactant concentration on drug solubilisation has not been investigated yet (Ahmed et al., 2003; Sultana et al., 2005, Tamanna et al., 2013). Similarly investigation regarding the effect of synergism on drug solubilisation has not been investigated as well.

But from pharmaceutical point of view synergism is a much practiced phenomenon especially in case of using excipients like antioxidants and antimicrobial preservatives. Because synergism gives an extra benefit of efficacy than is expected on the basis of their individual activities (Cater, 1987; Lieberman et al., 1989; Shiv, 1990). Surfactants are claimed to be pharmaceutical excipient and these may have synergistic effects on drug solubilisation which needs to be explored.

Polyvinylpyrrollidone K 30 (PVP K 30) and Polyethylene glycol 6000 (PEG 6000) are two mention worthy surfactants. PVP K 30 is a member of nonionic polymeric surfactant family called 'polyvinylpyrrollidones'and PEG 6000 is a member of an another nonionic polymeric surfactant family called 'polyoxyehtylene glycols'.

Characteristically these surfactants are non irritant and bio compatible. Moreover each of these surfactants has got pharmaceutical, medicinal and industrial values. It is reported that polyvinylpyrrollidones are widely used as formulation stabilizers and as drug solubilisers. Secondly these agents are used as thickeners and as wetting agents as well (A PhA, 1986; Lieberman et al., 1989). Note that wetting of water insoluble drugs in GIT is a prerequisite for rapid drug action. Whilst polyoxyehtylene glycols are highly recognized for their surfactant properties and beneficial medicinal uses. These agents retards mechanical haemolysis, changes the inhibition pattern of response activation and can be used for cancer therapy. In pharmaceutical fields these agents have been successfully used for wetting of hydrophobic drugs and for enhancing viscosity of pharmaceutical preparations. Thus uses of PEG 3350 (an another member of polyoxyehtylene glycols) in colonoscopy and in chronic constipation has been well documented (A PhA, 1986; Pashankar et al., 2003a,b; Sultana et al., 2012; Sultana et al., 2015). 
The same is true for PEG 20000 (an another member of polyoxyehtylene glycols) and Tween 80 (i.e Polysorbate 80). PEG 20000 and Tween 80 are also non ionic, non irritant and bio compatible surfactants of current use (A PhA, 1986; Lieberman, et al., 1988).

This present study therefore aimed at investigating the effects of concentration and synergism on drug solubilisation behavior of PVP K 30 and PEG 6000. Here concentration studies were planned to carry out at different values ranging from $2 \%$ to $10 \%$ and synergistic study was planned to carry out on different combinations made up with PVP K 30, PEG 6000, PEG 20000 and Tween 80 at the optimum concentration level. In the study theophylline was chosen as the sample drug. The rationale behind chosing theophylline was that this drug is a well established anti asthma agent that is in use over the past 50 years (as cited in Sultana, 1994). Moreover this drug has got an established metabolic profile and pharmacokinetic parameters (Hardman \& Limbird, 1996; Shahidi, 2015).

To the best knowledge of awareness of the authors such investigational study on solubilising behavior in terms of concentration and synergism of these non ionic surfactants using theophylline has not been done.

\section{MATERIALS AND METHOD}

Polyvinylpyrrollidone K 30 (Merck), Polyethylene glycol 6000 and Polyethylene glycol 20000 (Merck) and Tween 80 (Merck) were gifts from Pharmacy lab., Jahangirnagar university. Theophylline (anhyd.) was a gift from Square Pharmaceuticals.

Drug solubilisation study was conducted at normal day temperature following the method as described by Arnarson and Elworthy, (1980) where the model drug (a few mcg) was mixed with the aqueous surfactant solution $(10 \%, 5 \mathrm{~mL})$ for $48 \mathrm{~h}$ using a rotary mixture at a slow rpm. Assuming the state of equilibrium was attained, the mixture was centrifuged for 2 mins at a speed of $2000 \mathrm{rpm}$. A definite quantity of the clear, supernatant of the drug-surfactant dispersions was removed and following dilution with methanol, absorbance was determined using a UV/visible spectrophotometer (Shimadzu $160 \mathrm{1PC}$ ) at a wave length of $272 \mathrm{~nm}$. The amount of drug solubilised was determined from standard calibration curve drawn with absorbance versus six concentration values ranging from $1 \mathrm{mcg} / \mathrm{ml}$ to $20 \mathrm{mcg} / \mathrm{ml}$. In the study surfactant solutions viz $8 \%, 6 \%, 4 \%$, and $2 \%$ were made from $10 \%$ following dilution technique. For preparing surfactant combinations volume adjustment technique using $4 \%$ of respective surfactant solutions were used.

\section{RESULTS AND DISCUSSION}

In the present study effects of concentration and synergism on solubilisation behavior of PVP K 30 and PEG 6000 were investigated. Results have been shown in different figures (Fig. $2 \& 3$ ) and in Table 1. Our results stated that the relationship between the amount of drug solubilised by the surfactant and concentration was inverse. The amount solubilised 
at the lowest concentration was the highest and amount solubilised at the highest concentration was the lowest. Such inverse trend of relationship was evident for both the surfactants under investigation. For instances figure 2 represented the solubilisation behavior of PVP K 30 . Amount of drug solubilised at $2 \%$ was $4.79 \mathrm{mg} / \mathrm{ml}$ and at $10 \%$ was $0.46 \mathrm{mg} / \mathrm{ml}$ which was the lowest among other values. The same was true for PEG 6000 (Fig. 3) where it showed the highest amount of drug solubilisation at $2 \%$ and at $10 \%$ showed the least. Such results were new indeed and 2\% surfactant concentration value was chosen for further investigation.

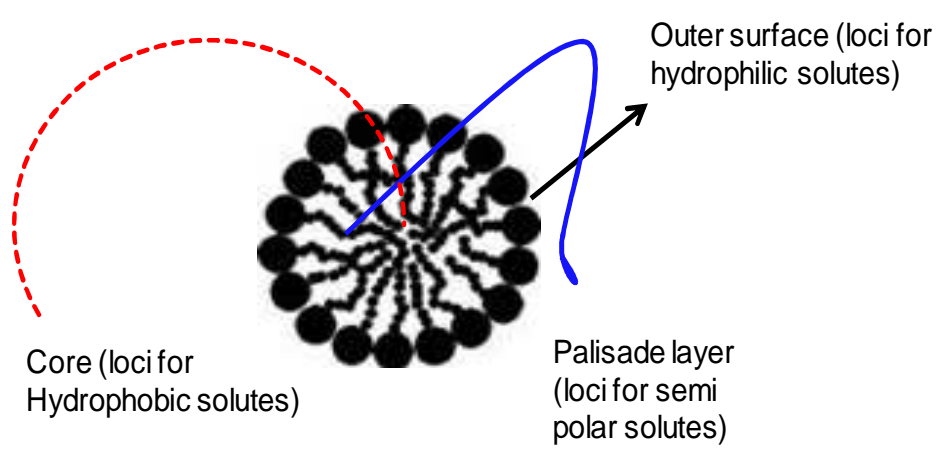

Fig. 1. A typical micelle with three regions

It was stated earlier that in our study effects of synergism on drug solubilisation behavior of PVP K 30 and PEG 6000 were investigated. For investigations not only the combination of PVP K 30 and PEG 6000 was used other combinations made up with PEG 20000 and Tween 80 were also used. In fact synergism is regularly practiced in pharmaceutical fields especially in case of using excipients like antioxidants and antimicrobial preservatives (Carter, 1987; Lieberman et al., 1989). Surfactants are claimed to be pharmaceutical excipients and they may have synergistic effects on drug solubilisation. So in this present study combinations of surfactants at $2 \%$ concentration level were used and solubilisation study were done using theophylline. In the study amount of drug solubilised by the surfactant combinations were compared to the amount solubilised by the corresponding surfactant used singly. Results have been shown in table 1 and our results failed to show synergism. For instances amount of drug solubilsed by PVP K 30 alone was $1.08 \mathrm{mg} / \mathrm{ml}$; while in presence of PEG 6000 the value was $9.39 \mathrm{mg} /$ $\mathrm{ml}$ and in presence of PEG 20000 and Tween 80 values were 9.67 and $9.40 \mathrm{mg} / \mathrm{ml}$ 
respectively. Similarly amount of drug solubilsed by PEG 6000 alone was $1.06 \mathrm{mg} / \mathrm{ml}$. In presence of PVP K 30, PEG 20000 and Tween 80 the values were 9.39, 9.43 and 9.37 $\mathrm{mg} / \mathrm{ml}$ respectively. Please note that amount of drug solubilsed by only Tween 80 and only PEG 20000 were 1.06 and $1.04 \mathrm{mg} / \mathrm{ml}$ respectively. Therefore our results clearly demonstrated that surfactant combinations under investigation were devoid of synergism when drug was theophylline.

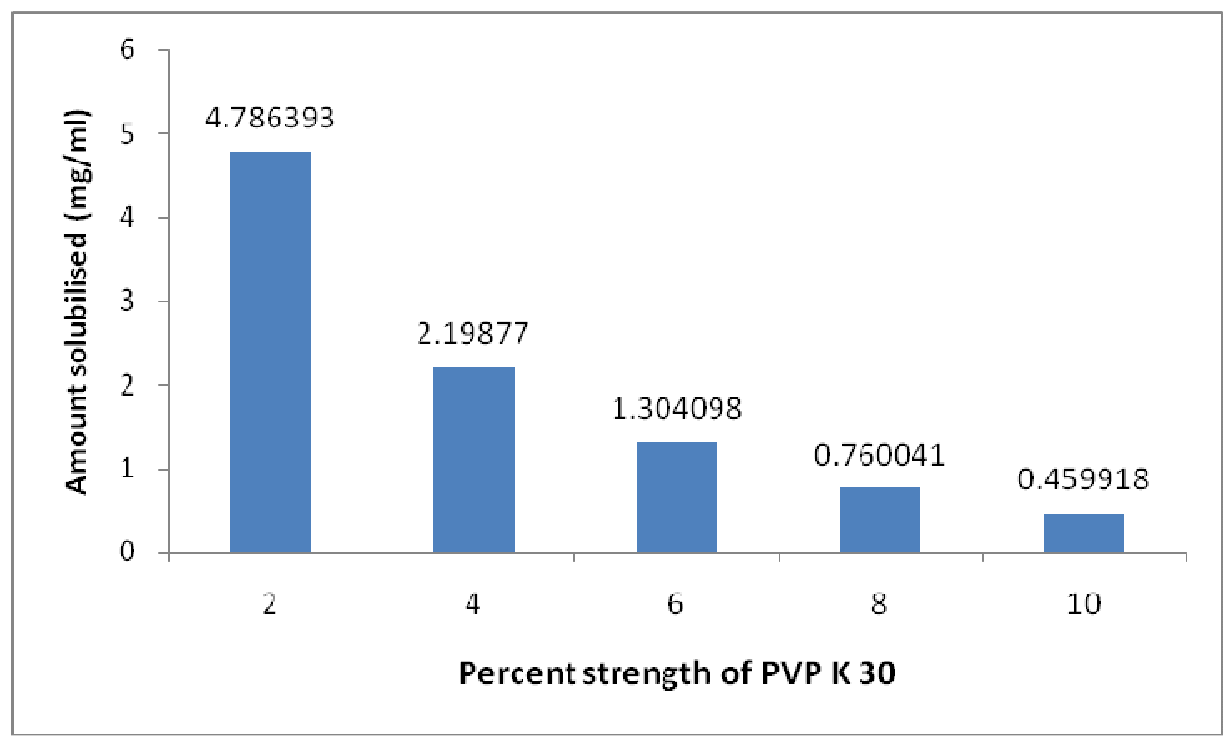

Fig. 2. Drug solubilisation in percent strength of PVP K 30 solution

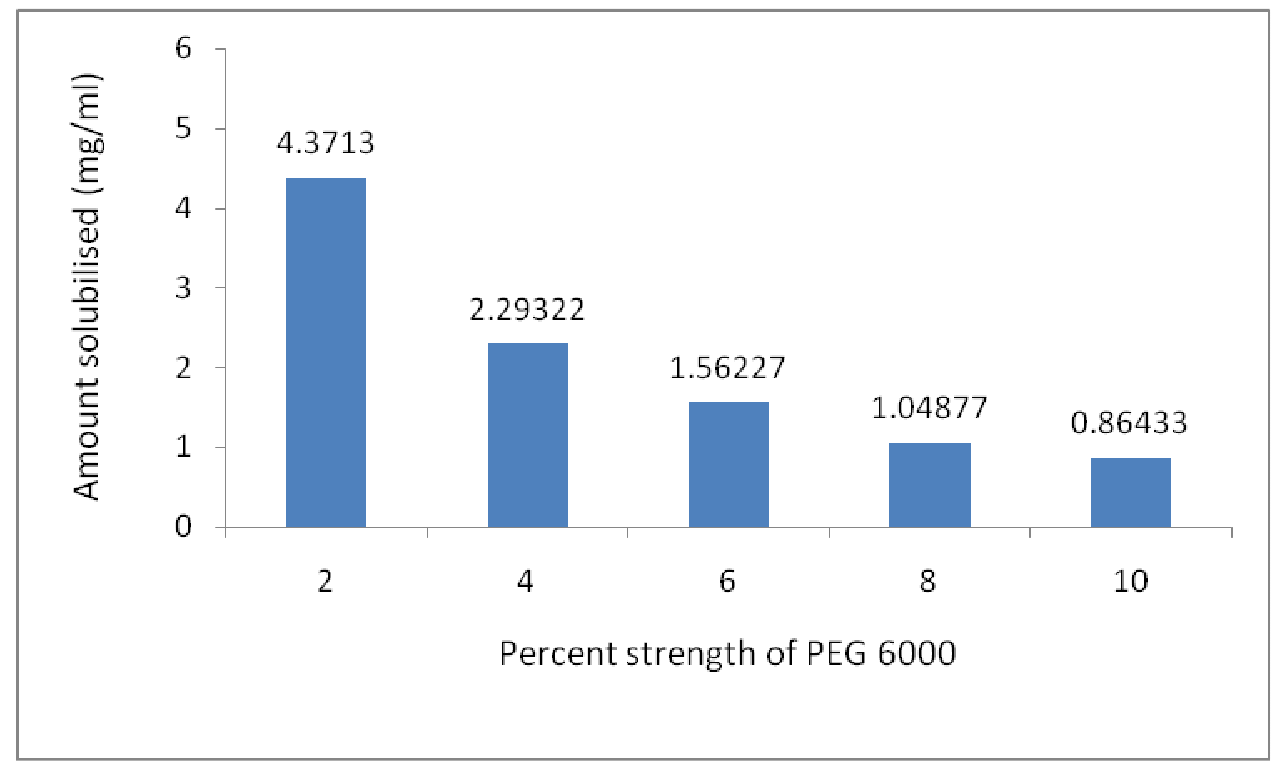

Fig. 3. Drug solubilisation in percent strength of PEG 6000 solution 
Table 1. Drug solubilisation in surfactant combinations

\begin{tabular}{l|l|l|l}
\hline Surfactants & $\begin{array}{l}\text { Solubilisation } \\
(\mathrm{mg} / \mathrm{mL})\end{array}$ & Surfactans in combinations & $\begin{array}{l}\text { Solubilisation } \\
(\mathrm{mg} / \mathrm{mL})\end{array}$ \\
\hline PVP K 30 & 1.08 & PVP K 30 + PEG 6000 & 9.39 \\
PEG 6000 & 1.06 & PVP K 30 + PEG 20000 & 9.67 \\
PEG 20000 & 1.04 & PVP K 30 + Tween 80 & 9.40 \\
Tween 80 & 1.06 & PEG 6000+ Tween 80 & 9.37 \\
Water & 1.09 & PEG 6000+ PEG 20000 & 9.43 \\
\hline
\end{tabular}

In our study tests were conducted with respect to water also. Here amount of drug solubilised by water was $1.09 \mathrm{mg} / \mathrm{ml}$ (Table 1). This value was not different to the values as obtained with PVP K 30 and PEG 6000; even with surfactant combinations under investigation. Such results were not the same as was expected. Such discrepancy in the results can be explained by the fundamentals of solubilisation phenomenon. It was stated earlier that solubilisation phenomenon involves many factors among which hydrophobicity of solute molecule is a major determinant. Therefore it might be that theophylline - the drug under investigation did not have enough hydrophobicity to be located inside the micellar core (Fig. 1); rather the drug might have hydrophilicity to be located on the outer surface of micelles and that ultimately decreased the extent of solubilsation. Here entrapment of drug molecule into the micellar core did not prevail and therefore solubilisation was not as was expected. This may suggest selection of drug might not be as wise as ought to be for drug solubilising study. This might be true as theophylline is a freely water soluble drug.

Conclusion: Our study thus concludes that for studying drug solubilisation behavior using PVP K 30 and PEG 6000 it is better to keep the working concentration of surfactant at $2 \%$ and secondly synergism is absent for combination of PVP K 30 and PEG 6000 and for their combinations containing PEG 20000 and Tween 80.

\section{REFERENCES}

A. PhA. 1986. Handbook of Pharmaceutical Excipients American Pharmaceutical Association and the Pharmaceutical Society of Great Britain.pp : 207, 217, 239.

Ahmed. J., Rahman. T. and Sultana, S. 2003. Comparison of drug solubilising behaviour between sodium lauryl sulphate and polyethylene glycol. Bangladesh Journal of Life sciences. 15(2), 151-157.

Arnarson, T. and Elworthy, P. H. 1980. Effects of structural variations of nonionic surfactants on micellar properties and solubilisation: surfactants based on erucyl behenyl $\left(\mathrm{C}_{22}\right)$ alcohols. J. Pharm. Pharmacol. 32, 381-385.

Carter, S. J. (ed). 1987. Cooper and Gunn's Dispensing for pharmaceutical students. $12^{\text {th }}$ edition. CBS publishers and Distributors. India. pp : 679.

Hardman, J. H. and Limbird, L. E (eds). 1996. Goodman and Gilman's The Pharmacological Basis of Therapeutics. $9^{\text {th }}$ edition. MacGraw - Hills, NY. pp : 673-675, 1786.

Liebermann, H. A., Rieger, M. M. and Banker, G. S. (eds). 1988. Pharmaceutical dosage forms. Disperse system. Volume I. Marcel Dekker Inc. Newyork and Basel. pp : 315-316, 319, $351-353$. 
Liebermann, H. A., Rieger, M. M. and Banker, G. S. 1989. Pharmaceutical dosage forms. Disperse system. Volume II. Marcel Dekker Inc. Newyork and Basel. pp : 199, 350 -354, 363

Pashankar, D. S., Bishop, W. P. and Loening-Baucke, V. 2003a. Long term efficacy of Polyethylene glycol for the treatment of chronic constipation in children with or without encopresis. J Clin Pediatr. 42(9), 815-819.

Pashankar, D. S., Bishop, W. P. and Loening-Baucke V. 2003b. Safety of Polyethylene glycol 3350 for the treatment of chronic constipation in children. Arch Pediatr Adolese Med. 157 (7), 661-664.

Rawlins, E. A. (eds). 2004. Betley's textbook of Pharmaceutics. $8^{\text {th }}$ edition. Bailliere Tindall. London. pp : 44, 51-59.

Shahidi, R. U. (eds). 2015. Quick Index of medical Products and Problems (QUIMP-17). Dhaka, Bangladesh.pp : 104-105.

Shiv, N, S. (ed). 1990. The technology of preparation and distribution of drugs and cosmetics. $1^{\text {st }}$ edition. Kislay Book House. India. pp : 38.

Sultana, S. 1994. Pharmacokinetic study of theophylline in pregnant rats. Masters thesis. Department of Pharmacy, School of Biomedical Sc, University of South Australia (Formerly known as SAIT).

Sultana, S. 2012. Evaluation of wetting property and purgation inducing capacity of nonionic surfactants in mice. Jahangirnagar University Journal of Biological Sciences. 1(1), 7780.

Sakina Sultana, Md. Ohidullah A H M Saifuddin and Saddam Hossain. 2015. Comparative studies of Hydration behavior and Density of PVP K 30, PEG 6000 and PEG 20000. Bangladesh Pharmaceutical journal. 18(1): 48-52

Tamanna, R., Jannatul, F., Faria, F. P. and Sakina, S. 2013. Capacity of non ionic and ionic surfactants for solubilisation of paracetamol. Bangladesh Pharmaceutical Journal. 16(1): 77-80. 